\title{
Three- and Four-jet Production at Low $x$ at HERA
}

\section{Jacek TURNAU*†}

Institute of Nuclear Physics, Krakow

E-mail: jacek.turnaudifj.edu.pl

\begin{abstract}
Three- and four-jet production is measured in deep-inelastic ep scattering at low $x$ and $Q^{2}$ with the $\mathrm{H} 1$ detector using an integrated luminosity of $44.2 \mathrm{pb}^{-1}$. Several phase space regions are selected for the three-jet analysis in order to study the underlying parton dynamics from global topologies to the more restrictive regions of forward jets close to the proton direction. The measurements of cross sections for events with at least three jets are compared to fixed order QCD predictions of $\mathscr{O}\left(\alpha_{s}^{2}\right)$ and $\mathscr{O}\left(\alpha_{s}^{3}\right)$ and with Monte Carlo simulation programs where higher order effects are approximated by parton showers. A good overall description is provided by the $\mathscr{O}\left(\alpha_{s}^{3}\right)$ calculation. Too few events are predicted at the lowest $x \sim 10^{-4}$, especially for topologies with two forward jets. This hints to large contributions at low $x$ from initial state radiation of gluons close to the proton direction and unordered in transverse momentum. The Monte Carlo program in which gluon radiation is generated by the colour dipole model gives a good description of both the three- and the four-jet data in absolute normalisation and shape.
\end{abstract}

European Physical Society Europhysics Conference on High Energy Physics

July 16-22, 2009

Krakow, Poland

\footnotetext{
${ }^{*}$ On behalf of the H1 Collaboration

${ }^{\dagger}$ This work was partially supported by Polish Ministry of Higher Education grant PBS/DESY/70/2006
} 


\section{Introduction}

Studies of the dynamics of long parton cascades at small values of $x$ at HERA have long history dating back to the early 90's when it became clear that inclusive measurements of DIS are not sufficient for finding limitations of the DGLAP approximation. The general strategy adopted for this task is to study hadronic final states in DIS, in particular to measure observables sensitive to the kinematic structure of gluon emissions. Within the DGLAP approach, only parton emissions ordered in transverse momentum are considered while BFKL accounts for non-ordered emissions.

Final states with jets in DIS are an ideal tool to investigate low $x$ dynamics: the jets can be used to tag higher order processes and furthermore provide direct access to the outgoing hard partons. The H1 and ZEUS measurements of forward jet production [1], 2] show that the leading order (LO) $\mathscr{O}\left(\alpha_{s}\right)$ calculations based on DGLAP greatly underestimate the data. The next-to-leading (NLO) $\mathscr{O}\left(\alpha_{s}^{2}\right)$ calculations can overcome some of the LO deficiencies, but the description remains unsatisfactory at low $x$ and $Q^{2}$. Here we present results of an analysis of events with at least three or four jets in the final state [3]. In contrast to more inclusive studies, i.e. jets and dijets, threeand four-jet final states require the radiation of at least one and two hard gluons, respectively, in addition to the $q \bar{q}$ pair from the dominating hard boson-gluon fusion scattering process $\gamma^{*} g \rightarrow q \bar{q}$. Of course this better control of underlying parton dynamics has its price: the requirement of at least three hard jets in the final state diminishes the phase space for additional parton emissions and thus the sensitivity to effects beyond DGLAP approximation.

Three-jet cross sections in DIS have been measured previously, both by the H1 [ [4] and the ZEUS [5, 6] collaborations. In these analyses the leading jets were required to have a large transverse momentum of at least $7 \mathrm{GeV}$. All measured cross sections were found to be well described by NLO $\mathscr{O}\left(\alpha_{s}^{3}\right)$ predictions in the collinear factorization scheme. The analysis on which we report here is performed in an extended phase space, covering jets with low transverse momenta down to $4 \mathrm{GeV}$, and based on a three times larger luminosity than used in the previous $\mathrm{H} 1$ publication [4]. The analysis reaches values of $x$ as low as $x=10^{-4}$. In addition, cross sections for events with at least four jets are measured for the first time in DIS.

The data are compared with the NLOJET++ fixed order calculation in the collinear factorization scheme, corrected for hadronisation effects. NLOJET++ is the only available program which provides perturbative calculations for jet cross sections in hadronic collisions to $\mathscr{O}\left(\alpha_{s}^{3}\right)$ accuracy. This corresponds to NLO and LO precision for the three- and four-jet cases, respectively. The two Monte Carlo generators RAPGAP and DJANGOH, which were able to describe reasonably well inclusive forward jet and dijet production at low $x$, are also tested. Both programs generate hard QCD $2 \rightarrow 2$ subprocesses (e.g. $\gamma^{*} g \rightarrow q \bar{q}$ ). Higher order QCD effects that produce further hard outgoing partons are generated in both RAPGAP and DJANGOH by parton showers: in RAPGAP the showers are ordered in the transverse momenta $\left(k_{T}\right)$ of the emissions, according to the DGLAP leading $\log Q^{2}$ approximation. DJANGOH uses the color dipole model (CDM), in which partons are generated by color dipoles, spanned between the partons in the cascade. Since the dipoles radiate independently, there is no $k_{T}$ ordering. For the hadronisation, the Lund string fragmentation is used both for RAPGAP and DJANGOH. 


\section{Results}

The analyzed data sample consists of events in the DIS region $0.1<y<0.7,10^{-4}<x<10^{-2}$ and $5 \mathrm{GeV}^{2}<Q^{2}<80 \mathrm{GeV}^{2}$, with at least 3 jets with $p_{T}^{*}>4 \mathrm{GeV}$ (in $\gamma^{*} p$ c.m.s.) and $-1<\eta_{\text {jet }}<$ 2.5 (in laboratory). The jets are selected to be asymmetric by imposing the condition $p_{T 1}^{*}+p_{T 2}^{*}>$ $9 \mathrm{GeV}$. The sample corresponds to integrated luminosity $44 \mathrm{pb}^{-1}$ and contains 384000 events with at least 3 jets. Figure 1 (left) presents the cross section for given number of jets, compared with
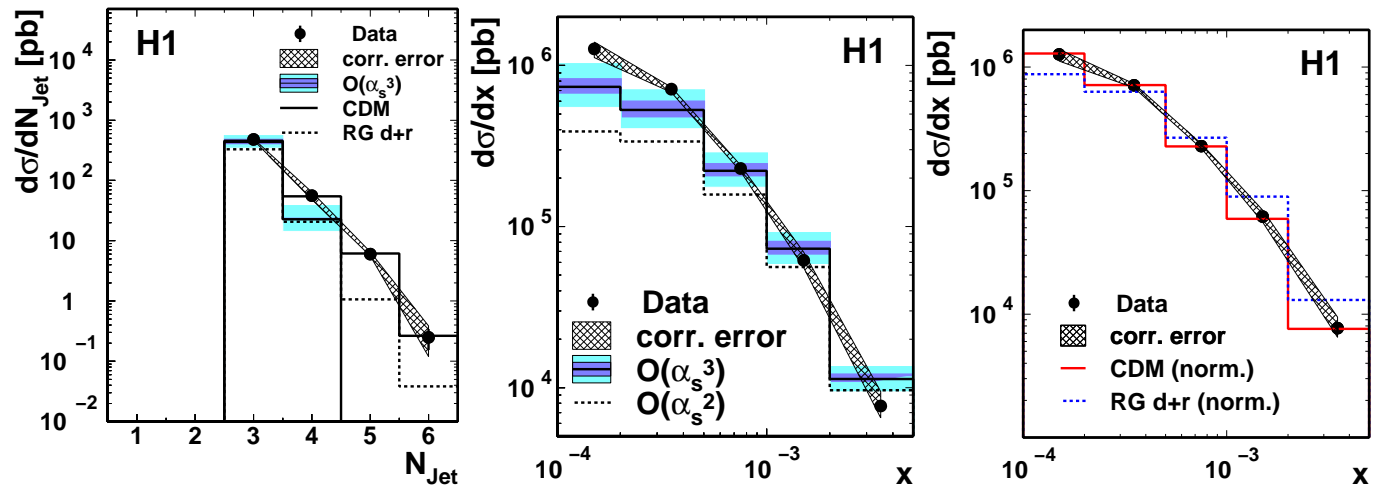

Figure 1: Differential cross sections as a function of the number of jets $N_{\text {jet }}$ found in the events and the Bjørken scaling variable $x$. The distributions are comapred to the NLO $\mathscr{O}\left(\alpha_{s}^{3}\right)$ prediction and the Monte Carlo programs RAPGAP and CDM.

predictions of the NLO $\mathscr{O}\left(\alpha_{s}^{3}\right)$ calculation and the Monte Carlo programs RAPGAP and CDM. The three-jet cross section is very well described by the NLO theory, while the 4-jet rate, predicted in LO, is underestimated by a factor 2.6. The CDM model provides an excellent description of the number of jets distribution up to 6 jets while the RAPGAP prediction underestimates the 4jet cross section by a factor 2.9. The $x$-distribution of the inclusive 3 -jet cross section (middle and right) is reasonably well described by the NLO $\mathscr{O}\left(\alpha_{s}^{3}\right)$ calculation except the region of very small $x<2 \cdot 10^{-4}$, where theory undershoots the data. This effect was either not observed or less accentuated in previous analyses with restricted phase space. The CDM description of the $x$ distribution is excellent apart from the normalization (factor 1.05). The RAPGAP Monte Carlo program fails both in normalization and shape.

In figure 2 we present illustrative examples of distributions describing the 3-jet system compared to the NLO $\mathscr{O}\left(\alpha_{s}^{3}\right)$ calculation. The theory describes the data well, apart from a slight deficit in normalisation (due to events with $>3$ jets) and a tendency to underestimate the jet cross section at forward pseudorapidities. In figure 3 we present illustrative examples of the data describing the 3-jet system compared to predictions of the RAPGAP and CDM programs. Again CDM provides a fairly good description of the data with exception of the jet transverse momentum distribution $p_{T}^{*}$. RAPGAP in most cases fails to describe the shape of the data. The observed excess of data versus QCD predictions in the region of forward jet rapidities and at low $x$ was investigated in further detail selecting subsamples with one or two forward jets. In the sample with one forward and two central jets ( $\mathrm{f}+2 \mathrm{c}$ ) we require two jets with $-1<\eta_{j e t}<1$ and one jet with $\eta_{j e t}>1.73$ and $x_{\text {jet }} \equiv \frac{E_{\text {jet }}}{E_{p, \text { beam }}}>0.035$. In the sample with two forward and one central jet $(2 \mathrm{f}+\mathrm{c}) 2$ jets are required 

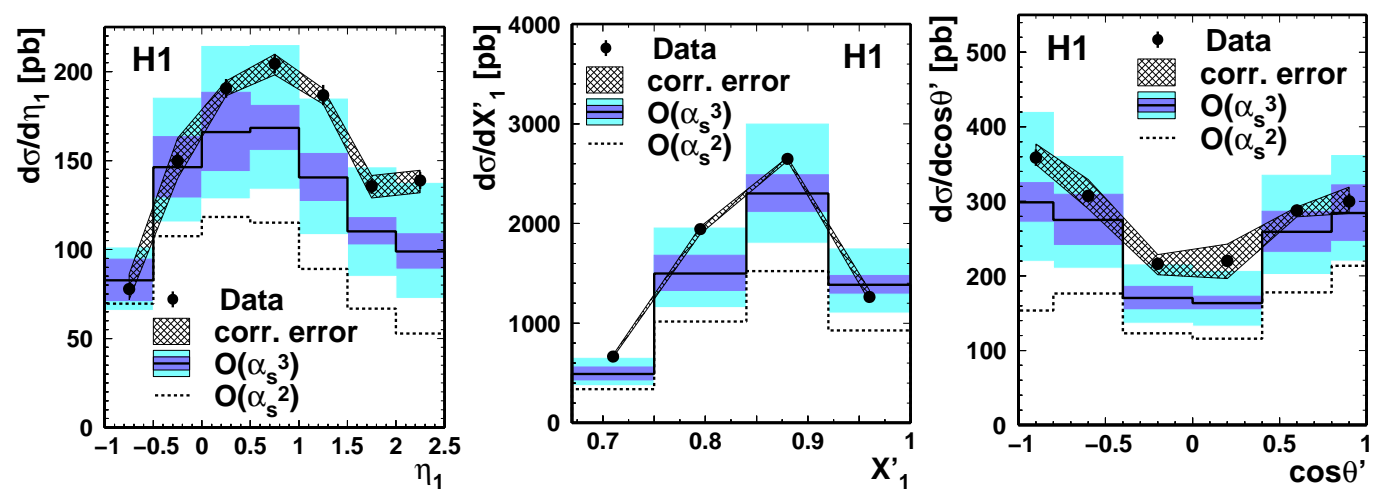

Figure 2: Differential cross sections as a function of pseudorapidity $\eta_{1}$ of the most energetic jet (left), scaled energy of this jet in the 3-jet c.m.s. (middle) and one of the angles defining the orientation of the 3 -jet sytem with respect to the virtual boson - parton plane (see [B] $)$. The distributions are compared to NLO $\mathscr{O}\left(\alpha_{s}^{3}\right)$.
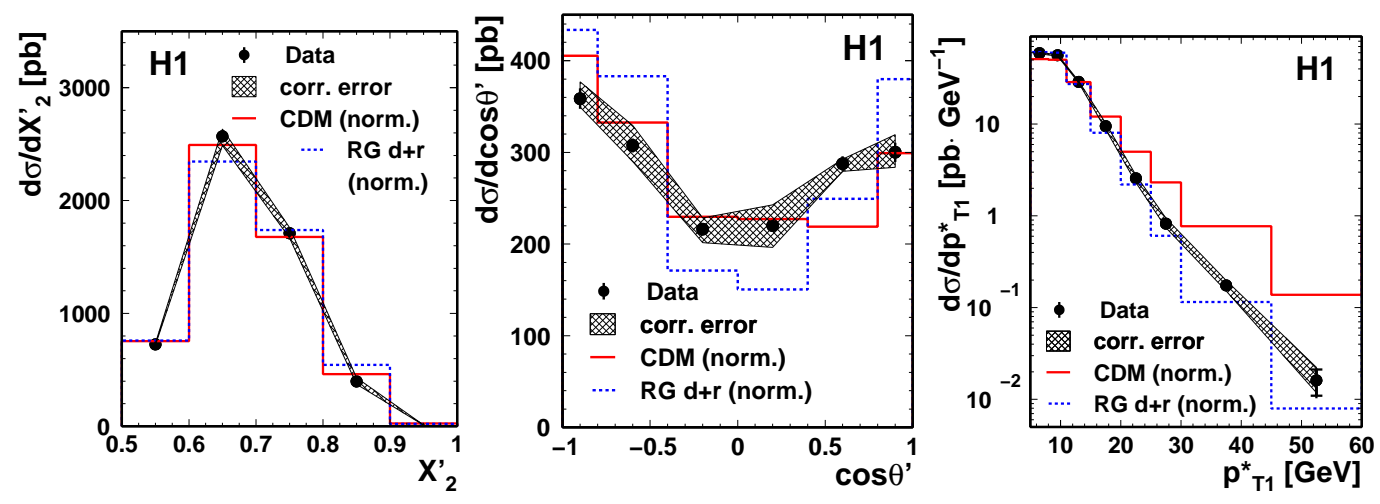

Figure 3: Differential cross sections as a function of the scaled energy of the second jet in the 3-jet c.m.s. (left), one of the angles defining the orientation of the 3-jet system (middle) and the transverse momentum of the first jet in the hadronic center of mass system. The distributions are compared to predictions of the RAPGAP and CDM generators.

with $\eta_{\text {jet }}>1$ and one central jet with $-1<\eta_{\text {jet }}<1$. In figure 4 the Bjorken $x$ distributions are presented for the $\mathrm{f}+2 \mathrm{c}$ (left) and $2 \mathrm{f}+\mathrm{c}$ (right) samples. The fixed order NLO $\mathscr{O}\left(\alpha_{s}^{3}\right)$ prediction gives a rather good description of the $\mathrm{f}+2 \mathrm{c}$ sample. At very low $x<2 \cdot 10^{-4}$ a deficiency of only $30 \%$ is observed. It should be noted that a much larger deficiency was observed in the inclusive forward jets study [1]]. This reflects the fact that at HERA three jets are already close to the limit of available phase leaving not much room for additional hard emissions. For the $2 \mathrm{f}+\mathrm{c}$ selection a dramatic change is observed at low $x$ from LO $\mathscr{O}\left(\alpha_{s}^{2}\right)$ to NLO $\mathscr{O}\left(\alpha_{s}^{3}\right)$ : the discrepancy at $x<2 \cdot 10^{-4}$ is reduced from a factor of 10 to 2.6. The large remaining deficiency exceeds the combined error of prediction and data and is thus highly significant. This data excess provides a strong hint for missing higher order QCD corrections. Note however, that the fraction of jets due to gluon radiation is expected to be larger for forward jets than for central jets, as confirmed by a study of the parton composition of three-jets in the CDM simulation. Thus a large fraction of the $2 \mathrm{f}+\mathrm{c}$ sample corresponds effectively to 4 -jet process for which the $\left(\alpha_{s}^{3}\right)$ calculation can only provide a leading 

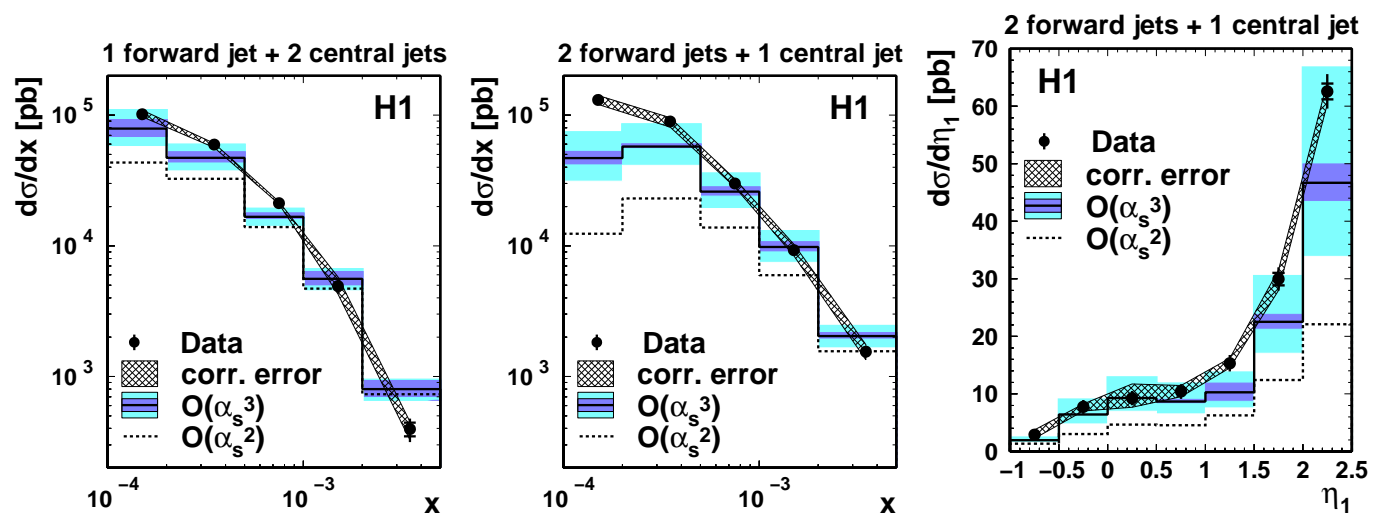

Figure 4: Differential cross sections as a function of the Bjorken scaling variable $x$ for the sample with one forward jet and two cental jets $(\mathrm{f}+2 \mathrm{c})$ (left), the sample with two forward jets and one cental jet $(2 \mathrm{f}+\mathrm{c})$ (middle). On the right differential cross section as a function of the pseudorapidity of the leading jet for the $2 \mathrm{f}+\mathrm{c}$ sample.

order perturbative estimate. Apart from small $x$ region $2 \mathrm{f}+\mathrm{c}$ sample is reasonably well described by NLO $\mathscr{O}\left(\alpha_{s}^{3}\right)$ calculation, example shown in figure $\emptyset$ (right).

A subsample of events with four or more jets was also studied. As already mentioned the NLOJET $++\mathscr{O}\left(\alpha_{s}^{3}\right)$ calculation can only provide a LO prediction for the final state with four jets. Thus comparisons of the measured four-jet cross sections were restricted to the CDM and RAPGAP predictions. CDM gives a very good description of all measured distributions (not shown here) including their normalization, except the differential cross section as a function of the leading jet transverse momentum. RAPGAP fails to describe shape and normalization of the differential distributions.

We conclude that remarkable result of the presented analysis is the success of the NLO $\mathscr{O}\left(\alpha_{s}^{3}\right)$ calculation for the cross sections with at least three jets. A huge improvement w.r.t. $\mathscr{O}\left(\alpha_{s}^{2}\right)$ theory is observed, especially for large positive jet rapidities and small values of Bjorken $x$. However there are regions of phase space where the fixed order NLO DGLAP $\mathscr{O}\left(\alpha_{s}^{3}\right)$ calculation can not describe the data. The LO matrix element with parton showers unordered in transverse momentum modelled by the Color Dipol Model describes the data for $\geq 3$ and $\geq 4$ jets surprisingly well. The $k_{T}$-ordered parton shower modelled by RAPGAP fails to describe the data, both in shape and normalisation.

\section{References}

[1] A. Aktas et al. [H1 Collaboration], Eur. Phys. J. C 46 (2006) 27 [arXiv:hep-ex/0508055].

[2] S. Chekanov et al. [ZEUS Collaboration], Phys. Lett. B 632 (2006) 13 [arXiv:hep-ex/0502029].

[3] F. D. Aaron et al. [H1 Collaboration], Eur. Phys. J. C 54 (2008) 389 [arXiv:0711.2606 [hep-ex]].

[4] C. Adloff et al. [H1 Collaboration], Phys. Lett. B 515 (2001) 17 [arXiv:hep-ex/0106078].

[5] S. Chekanov et al. [ZEUS Collaboration], Eur. Phys. J. C 44 (2005) 183 [arXiv:hep-ex/0502007].

[6] S. Chekanov et al. [ZEUS Collaboration], Nucl. Phys. B 786, 152 (2007) [arXiv:0705.1931 [hep-ex]]. 